\title{
DYNAMICS OF GAS BUBBLES IN WHOLE BLOOD AND PLASMA* $\dagger$
}

\author{
WEN-JEI YANG \\ Department of Mechanical Engineering. University of Michigan. Ann Arbor. Mich. 48104. \\ U.S.A.
}

\begin{abstract}
Analytical solutions are obtained for both the radius-time relation and the rate of solution by diffusion of a gas bubble in plasma and whole blood with reduced hemoglobin. If an oxygen bubble is situated in the blood. a concentration boundary is formed in which the combined processes of diffusion and chemical reaction takes place. The effect of surface tension on the bubble dynamics is also considered. Applications of the analytical results include the extracorporeal oxygenation of the blood and the determination of the diffusion coefficient in plasma and the reaction velocity constant in whole blood.
\end{abstract}

\section{INTRODUCTION}

THE DISSOLUTION of gas bubbles in a liquidgas solution with simultaneous chemical reaction between the dissolved gas and the liquid is of concern in several problems of biological and physical interest. Among them may be mentioned the dissolution of oxygen bubbles in blood in extra-corporeal circulation during open heart surgery. and that of carbon dioxide bubbles in water.

When an oxygen bubble is situated in whole blood the oxygen gas diffuses across the bubble surface into the blood and immediately combines with the undersaturated hemoglobin. Only a small fraction of the oxygen gas is dissolved in the plasma. The chemical reaction between the oxygen and the hemoglobin takes place in the concentration boundary layer. which grows in thickness over the bubble surface. The combined processes of diffusion and second-order chemical reaction can be described by the following differential equation (Forster. 1964).

$\frac{\partial\left[\mathrm{O}_{2}\right]}{\partial t}=\mathscr{R} \Gamma^{2}\left[\mathrm{O}_{2}\right]+k^{\prime \prime}\left[\mathrm{HbO}_{2}\right]-k^{\prime}\left[\mathrm{O}_{2}\right][\mathrm{Hb}]$

where $t$ is time, $\nabla^{2}$ is the Laplacian operator:
$\left[\mathrm{O}_{2}\right] .[\mathrm{Hb}]$ and $\left[\mathrm{HbO}_{2}\right]$ are the concentrations of $\mathrm{O}_{2}$. reduced hemoglobin. and oxygenated hemoglobin. respectively. The symbol $k^{\prime \prime}$ is the reaction velocity constant for the dissociation of $\mathrm{O}_{2}$ from $\mathrm{HbO}_{2}: k^{\prime}$ is the reaction constant for the association of $\mathrm{O}_{2}$ and $\mathrm{Hb}$ to form $\mathrm{HbO}_{2}, \mathscr{Z}$ is the diffusion coefficient. When the chemical constituents are not in equilibrium, a second equation is required to define the relationships among the reactants (Forster, 1964 and Gibson. 1959).

$$
\frac{\partial\left[\mathrm{HbO}_{2}\right]}{\partial t}=-k\left[\mathrm{HbO}_{2}\right]+k^{\prime}[\mathrm{Hb}]\left[\mathrm{O}_{2}\right] .
$$

Forster (1964) has presented a comprehensive survey of the literature pertinent to the problems of gas uptake by red cells as described by equations (1) and (2). It is summarized as follows.

Numerical solutions of equations $(1)$ and (2) have been obtained for several cases: (a) Diffusion of a liquid gas into and out of an infinite sheet of hemoglobin solution. the onedimensional case with and without a membrane (containing no hemoglobin) on the surface. by Klug et al. (1956) and Nicolson et al. (1951). Equations (1) and (2) were solved by

* Received 30 March 1970.

+ The work was supported by a grant from the National Institute of Health. 
numerical methods involving finite differences and successive approximations. (b) Diffusion of liquid gas into spherical and discoidal volumes (three-dimensional) containing no hemoglobin. This was done by Forster et al. (1959) through the use of a digital computer.

In this paper, the simultaneous diffusion and chemical reaction process is investigated for the case where a gas contained in a spherical bubble diffuses into whole blood. Consider the whole blood as a homogeneous mixture of plasma and hemoglobin, and let equation (1) describe the diffusion of a gas from a bubble into the whole blood accompanied by chemical reaction. It is assumed that only the association of the dissolved gas and the reduced hemoglobin takes place. In other words, the reaction products such as oxygenated hemoglobin are constantly removed from the immediate vicinity of the gas bubble. As a result, no dissociation process occurs and the gas bubble is surrounded by the reduced hemoglobin at a constant concentration. The postulation is reasonable for two cases: (1) When the gas bubble is situated in a moving blood stream. Then a relative motion occurs between the bubble and the blood. resulting from their bulk motions and the motion of the bubble surface due to its shrinkage. (2) During the initial stage of bubble collapse in the quiescent blood. Under the above assumption, equation (1) may be modified to the form

$$
\frac{\mathrm{D}\left[\mathrm{O}_{2}\right]}{\mathrm{D} t}=\mathscr{D} \nabla^{*}\left[\mathrm{O}_{2}\right]-K\left[\mathrm{O}_{2}\right]
$$

where $K$ is $k^{\prime}\left[\mathrm{O}_{2}\right]$ and $\mathrm{D} / \mathrm{D} t$ is the substantial derivative including both the local and convective effects on diffusion. Equation (3) can be solved for the concentration from which the bubble radius-time relationship and the rate of mass diffusion of the gas from the bubble can be determined. In plasma where chemical reaction is absent $(K=0)$, analytical results are general, i.e. free of the restriction imposed by the above postulation.

\section{ANALYSIS}

Consider that at the initial time a spherical gas bubble of radius $R_{0}$ is situated in plasma or whole blood, in which the concentration of the dissolved gas is uniform and equal to $C_{\infty}$. Let the liquid (referring to plasma or whole blood) be at constant temperature and pressure, and the dissolved gas concentration for a saturated liquid at this temperature and pressure be denoted by $C_{s}$. The origin of a spherical polar coordinate system is fixed at the center of the gas bubble which is at rest. At any time $t$ when the bubble radius is $R$, the diffusion of the gas across the bubble surface into the liquid is accompanied by simultaneous chemical reaction of first order in the liquid. The dissolved gas concentration $C$ at a point in the liquid at a distance $r$ from the origin, is governed by the diffusion equation (3). It is rewritten in spherical coordinates as

$$
\frac{\partial \mathrm{C}}{\partial t}=\mathscr{D} \nabla^{2} \mathrm{C}-K C
$$

where $K>0$ for whole blood, while $K=0$ in the case of plasma. The motion of the bubble surface resulting from the shrinkage of the bubble introduces convective effects into the diffusion process. The convective term has been omitted from the LHS of equation (4). The resulting approximation is good so. long as (1) the concentration of dissolved gas in the liquid surrounding the bubble is much smaller than the gas density in the bubble. and (2) the region in the solution around the bubble through which the diffusion process takes place is soon much larger than the bubble itself. The first physical reason is valid especially in the presence of association process, while the other reason will be confirmed later by numerical results.

The appropriate initial and boundary conditions are

$$
\begin{aligned}
& C(r, 0)=C_{\infty} \\
& C(\infty, t)=C_{\infty}, \quad C(R, t)=C_{s} .
\end{aligned}
$$


In the absence of chemical reaction, i.e. surface is

$K=0$, the solution $v(r, t)$ of equation (4). subject to the conditions (5) and (6), may be solved through the introduction of the new variable

$$
u=r\left(v-C_{s}\right)
$$

and

$$
\xi=r-R
$$

The solution of the problem, which is identical with a familiar problem in heat conduction. is found to be

$$
\begin{aligned}
v(r, t)=C_{\infty}-\frac{R\left(C_{s}-C_{\alpha}\right)}{r} & {\left[1-\frac{2}{\pi} \int_{0}^{\infty}\right.} \\
\left.\mathrm{e}^{-\lambda_{\lambda} \lambda_{t}} \frac{\sin [(r-R) \lambda]}{\lambda} \mathrm{d} \lambda\right] & \text { (9) }
\end{aligned}
$$

If $v(r . t)$ is the solution for the case $K=0$ and the same boundary conditions, it may be verified by differentiation that

$$
C(r, t)=C_{\alpha}+K \int_{0}^{t} \mathrm{e}^{-K^{\prime}} v\left(r, t^{\prime}\right) \mathrm{d} t^{\prime}+v(r, t) \mathrm{e}^{-K t}
$$

satisfies equation ( 4 ) and the boundary conditions. The substitution of equation (9) into equation (10) produces

$$
\begin{aligned}
C(r . t)= & C_{\alpha}+\frac{R\left(C_{s}-C_{\infty}\right)}{r} \mathrm{e}^{-K^{\prime} t} \\
& {\left[1-\frac{2}{\pi} \int_{0}^{\infty} \mathrm{e}^{-\lambda^{2} t^{\prime}} \frac{\sin [(r-R) \lambda]}{\lambda} \mathrm{d} \lambda\right] } \\
& +K \int_{0}^{t} \mathrm{e}^{-\kappa^{\prime} \frac{R\left(t^{\prime}\right)\left(C_{s}-C_{\infty}\right)}{r}} \\
& {\left[1-\frac{2}{\pi} \int_{0}^{\infty} \mathrm{e}^{-\infty \lambda^{\prime} t} \frac{\sin [(r-R) \lambda]}{\lambda} \mathrm{d} \lambda\right] \mathrm{d} t^{\prime} . }
\end{aligned}
$$

The concentration gradient at the bubble

Thus, the rate of mass flow into the liquid has the value

$$
\begin{aligned}
\frac{\mathrm{d} m}{\mathrm{~d} t}= & 4 \pi R^{2} \mathscr{R}\left(\frac{\partial C}{\partial r}\right)_{r=R}=4 \pi R^{2} \mathscr{D}\left(C_{s}-C_{\alpha}\right) \\
& \times\left(\frac{1}{R}+\frac{\mathrm{e}^{-K t}}{\sqrt{\pi \mathscr{D} t}}+\sqrt{\frac{K}{\mathscr{Q}}} \operatorname{erf} \sqrt{K t}\right) .
\end{aligned}
$$

Or, in dimensionless form as

$$
\frac{\mathrm{d} m^{*}}{\mathrm{~d} t^{*}}=4 \pi\left(R^{*}\right)^{2}\left(\frac{\partial C^{*}}{\partial r^{*}}\right)_{r^{*}=R^{*}} C_{P}^{*}=4 \pi\left(R^{*}\right)^{2}
$$

$\times C_{p}^{*}\left(C_{\alpha}^{*}-1\right)\left(\frac{1}{R^{*}}+\frac{\mathrm{e}^{-K^{*} t^{*}}}{\sqrt{\pi t^{*}}}+\sqrt{K^{*}} \operatorname{erf} \sqrt{K^{*} t^{*}}\right)$

where

$$
m^{*}=\frac{m}{\rho_{g} R_{0}{ }^{3}}, I^{*}=\frac{\mathscr{D} t}{R_{0}{ }^{2}}, R^{*}=\frac{R}{R_{0}}, C^{*}=\frac{C}{C_{s}} .
$$

$r^{*}=\frac{r}{R_{0}}, C_{\rho}^{*}=\frac{C_{s}}{\rho_{g}}, C_{\propto}^{*}=\frac{C_{\alpha}}{C_{s}}, K^{*}=\frac{K R_{0}{ }^{2}}{\mathscr{L}}$

However, the rate change of the mass of gas inside the bubble is

$$
\frac{\mathrm{d} m}{\mathrm{~d} t}=4 \pi R^{2} \rho_{\eta} \frac{\mathrm{d} R}{\mathrm{~d} t} .
$$

Hence, the equality of equation (13) and (16) gives the differential equation for the bubble radius in dimensionless form as

$$
\begin{aligned}
\frac{\mathrm{d} R^{*}}{\mathrm{~d} t^{*}}=-C_{\boldsymbol{\rho}}^{*}\left(1-C_{\propto}^{*}\right) & \left(\frac{1}{R^{*}}+\frac{\mathrm{e}^{-K^{*} t^{*}}}{\sqrt{\pi t^{*}}}\right. \\
& \left.+\sqrt{K^{*}} \operatorname{erf} \sqrt{K^{*} t^{*}}\right) .
\end{aligned}
$$


The last equation is integrated to yield the radius-time relationship for the bubble

$$
\begin{aligned}
1-\left(R^{*}\right)^{2} & =\frac{1}{2} C_{\rho}^{*}\left(1-C_{\infty}^{*}\right)\left(t^{*}+\int_{0}^{t^{*}} \frac{R^{*} \mathrm{e}^{-K^{*} t^{*}}}{\sqrt{\pi t^{*}}}\right. \\
d t^{*} & \left.+\sqrt{K^{*}} \int_{0}^{t^{*}} R^{*} \operatorname{erf} \sqrt{K^{*} t^{*}} \mathrm{~d} t^{*}\right) \cdot
\end{aligned}
$$

Effect of surface tension on diffusion and bubble dynamics

The force balance on the bubble surface requires that

$$
p+\frac{2 \sigma}{R}=p_{00}\left(\frac{\rho_{g}}{\rho_{g 0}}\right)^{n}
$$

where $p$ and $p_{g}$ are respectively the liquid and gas pressures exerted on the bubble surface, $\sigma$ is the surface tension, $\rho_{g}$ is the gas density, $n$ is the polytropic exponent, and the subscript 0 indicates the physical state corresponding to $t \leqslant 0$. Let $\rho_{\text {goo }}$ be the density of the gas under the same conditions of pressure and temperature. with a gas-liquid surface of zero curvature. Then the thermodynamic equation of state for an ideal gas gives

$$
p=\rho_{g \infty} \frac{\bar{R}_{g}}{M} T
$$

where $\vec{R}_{g}$ is the universal gas constant, $M$ is the molecular weight of the gas and $T$ is the liquid temperature. The combination of equations (19) and (20) followed by the substitution of $\rho_{g 0}=p_{g 0} M / \bar{R}_{g} T$ yields

$$
\rho_{g}=\rho_{g 0} \alpha^{1 / n}
$$

where

$$
\begin{aligned}
\alpha & =\rho^{*}+\frac{\sigma^{*}}{R^{*}}, \rho^{*}=\frac{\rho_{g \infty}}{\rho_{g 0}}, \sigma^{*}=\frac{2 \sigma}{R_{0} p_{g 0}} \\
& =\frac{2 \sigma M}{\bar{R}_{g} \rho_{g 0} T R_{0}} .
\end{aligned}
$$

Hence, the mass of gas in the bubble is

$$
m=\frac{4 \pi}{3} R_{0}{ }^{3} \rho_{g v} \alpha^{1 / n}
$$

so that

$$
\frac{\mathrm{d} m^{*}}{\mathrm{~d} t^{*}}=4 \pi\left(R^{*}\right)^{2} \frac{\mathrm{d} R^{*}}{\mathrm{~d} t^{*}} \alpha^{\mathrm{y} / n}\left(1-\frac{\sigma^{*}}{3 R^{*} n \alpha}\right) .
$$

The equality of (13) and (23) gives

$$
\begin{aligned}
\frac{\mathrm{d} R}{\mathrm{~d} t}= & \frac{\mathscr{D}\left(C_{\infty}-C_{s}\right)}{\rho_{g 0} \alpha^{1 / n}\left(1-\sigma^{*} / 3 R^{*} n \alpha\right)} \\
& \left(\frac{1}{R}+\frac{\mathrm{e}^{-K t}}{\sqrt{\pi \mathscr{D} t}}+\sqrt{\frac{K}{\mathscr{D}}} \operatorname{erf} \sqrt{K t}\right) .
\end{aligned}
$$

When surface tension is neglected. both $C_{s}$ and $\rho_{g}$ remain constant throughout the entire process of bubble collapse. On the other hand, when the effect of surface tension is included, both $C_{s}$ and $\rho_{g}$ vary as a function of the instantaneous bubble size. However, it can be shown that their ratio $C_{\rho}^{*}$ is not affected by surface tension effects. $C_{s}$ may be expressed as

$$
C_{s}=C_{p}^{*} \rho_{00} \alpha^{1 / n}
$$

$C_{\infty}$ is conveniently expressed in terms of $C_{3}^{*}$ by the relationship $C_{\infty}=C_{s}^{*} C_{\infty}^{*} \rho_{g \infty}$. Equation (24) can now be written in dimensionless form as

$$
\begin{array}{r}
\frac{\mathrm{d} R^{*}}{\mathrm{~d} t^{*}}=-\frac{C_{\rho}^{*}\left(\alpha^{1 / n}-\rho^{*} C_{x}^{*}\right)}{\alpha^{1 / n}\left(1-\sigma^{*} / 3 R^{*} n \alpha\right)}\left(\frac{1}{R^{*}}+\frac{\mathrm{e}^{-K t^{*}}}{\sqrt{\pi t^{*}}}\right. \\
\left.+\sqrt{K^{*}} \text { erf } \sqrt{K^{*} t^{*}}\right) .
\end{array}
$$

Take a special case where the gas inside the bubble undergoes an isothermal process during the collapse of the bubble. This corresponds to the case where $n=1$ and $\rho^{*}=1$ or $\alpha=1+\sigma^{*} / R^{*}$. Equations (23) and (26) may now be written as

$$
\frac{\mathrm{d} m^{*}}{\mathrm{~d} t^{* *}}=4 \pi\left(R^{*}\right)^{2} \frac{\mathrm{d} R^{*}}{\mathrm{~d} t^{*}}\left(1+\frac{2}{3} \frac{\sigma^{*}}{R^{*}}\right)
$$

and

$$
\begin{aligned}
\frac{\mathrm{d} R^{*}}{\mathrm{~d} t^{*}}=-C_{\rho}^{*} \frac{1-C_{x}^{*}+\left(\sigma^{*} / R^{*}\right)}{1+} & \left(\frac{1}{s^{*}\left(\sigma^{*} / R^{*}\right)}+\frac{\mathrm{e}^{-\kappa^{*} t^{*}}}{\sqrt{\pi t^{*}}}\right. \\
& \left.+\sqrt{K^{*}} \operatorname{erf} \sqrt{K^{*} t^{*}}\right)
\end{aligned}
$$

respectively 
THEORETICAL RESULTS AND DISCUSSION

An examination of equations (14). (18), (23). (26). (27) and (28) has revealed that (1) When the effect of surface tension is neglected. both the diffusion of the dissolved gas in the liquid and the radius-time relation for dissolving bubbles are functions of four dimensionless parameters $C_{0}^{*} . C_{*}^{*} . K^{*}$ and $t^{*}$. (2) When the effect of surface tension is taken into consideration. both the diffusion and the radius-time relation become functions of seven dimensionless parameters $C_{\rho}^{*}, C_{x}^{*}$. $K^{*} \cdot f^{*}, \sigma^{*}, \rho^{* *}$ and $n$. The parameters $\rho^{*}$ and $n$ depend on the thermodynamic process of the gas inside the bubble during bubble collapse. For an isothermal process. both $\rho$ and $n$ are unity and consequently only the remaining five parameters influence the diffusion and the bubble dynamics. It must be noted that because the diffusion cocfficients of plasma and whole blood are not available. that of water has been employed in the follow: ing calculations.

Equation (11) is numerically integrated for $C_{x}=0$ using a digital computer. The results for the concentration-time relation are graphically illustrated in Fig. 1 . It is shown in the figure that for both plasma $\left(K^{*}=0\right)$ and whole blood $\left(K^{*}=4880\right.$, corresponding to $K=9.76 \mathrm{sec}^{-1} . \alpha=2 \times 10^{-5} \mathrm{~cm}^{2} / \mathrm{sec}$ and $R_{0}=0.1 \mathrm{~cm}$ for example) concentration boundary layers in the solution around the

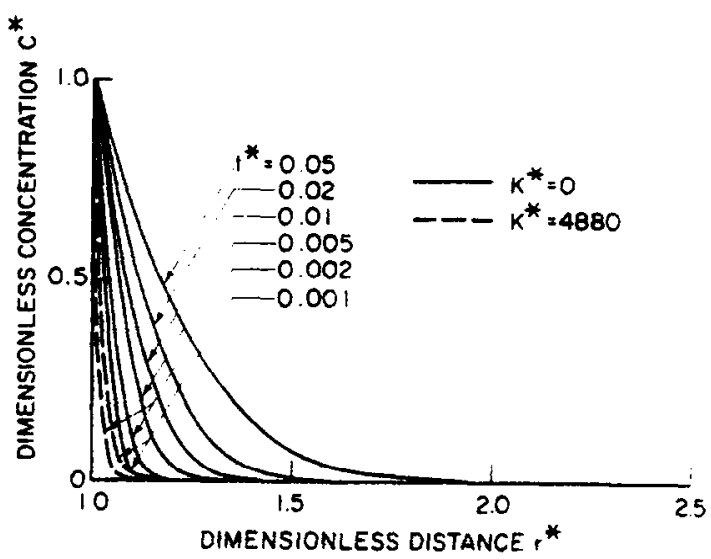

Fig. 1. Concentration-time relation for dissolved gas in whole blood $\left(K^{*}=4880\right)$ and plasma $\left(K^{*}=0\right)$ for $C_{y}^{*}=0$.

bubble through which the diffusion takes place become larger than the bubble itself for small times. This observation confirms the second condition under which the omission of the convective term in equation (4) is justified.

The numerical results of equation (18) are obtained through the use of a digital computer for two distinct cases: The case of $K^{*}=0$ and $C_{x}^{*}=0$ for plasma is shown in Fig. 2 and the case of $C_{0}^{*}=0.025$ (corresponding to $C_{\mathrm{s}}=3.26 \times 10^{-5} \mathrm{~g} / \mathrm{cm}^{3}$ and $\rho_{\mathrm{g} 0}=1.307 \times 10^{-3}$ $\left.\mathrm{g} / \mathrm{cm}^{3}\right)$ and $C_{\alpha}^{*}=0$ for whole blood is depicted in Fig. 3. Figure 2 indicates that the time required for a gas bubble to dissolve completely in the plasma is shortened as the value of $C_{p}^{*}$ increases. i.e. as $C_{s}$ is increased and/or

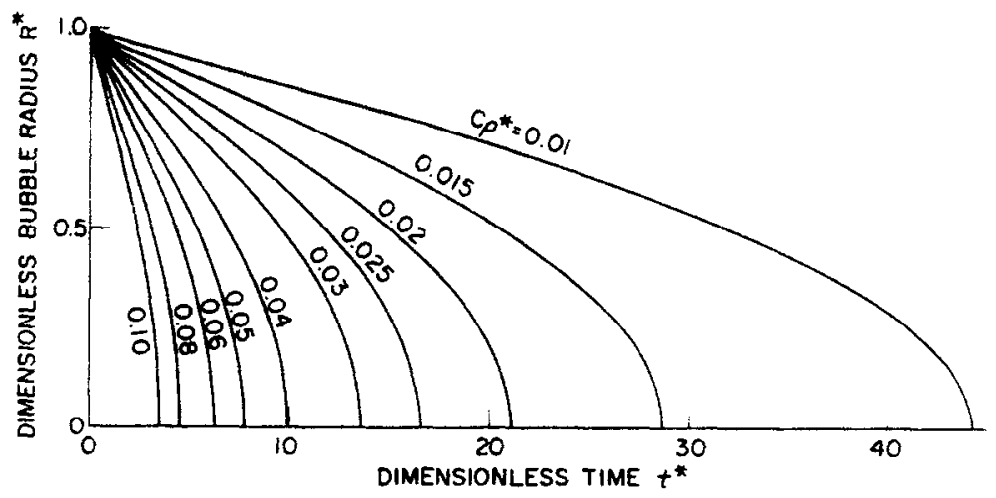

Fig. 2 Radius-time relation for dissolving bubbles in plasma $(K=0)$ for $C:=0$. 


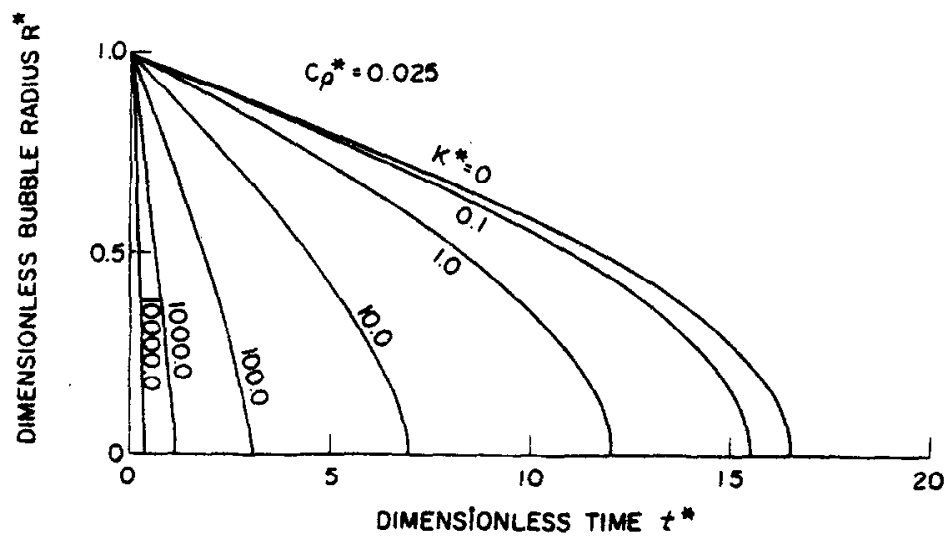

Fig. 3. Radius-time relation for dissolving bubbles in whole blood for $C_{p}^{*}=0.025$ and $C_{*}^{*}=0$.

$\rho_{g}$ is decreased. This means that at the same temperature at saturation, the bubble of a gas with lower density may be completely dissolved in the plasma in less time than that of a gas with higher density.

Figure 3 illustrates that as the magnitude of the reaction velocity constant $K^{*}$ is increased. the time of complete solution of a gas bubble in the whole blood is shortened. This observation can be easily explained physically. Since the term - KC on the RHS of equation (4) signifies the existence of a uniformly distributed mass sink of strength $K C$ in the liquid region; the larger the value of $K$, the stronger is the strength of the mass sink and consequently the diffusion is enhanced. This results in a faster dissolution of a gas bubble in the liquid.

Owing to the mathematical complication of equaton (26) or (28), the effect of surface tension on the diffusion and bubble collapse will be examined using an approximate solution obtained for large times. As indicated by the slope of the $R^{*}$ vs. $t^{*}$ curves in Figs. 2 and 3 , significant diffusion takes place when enough time has elapsed. Consider the most practical case. where the gas contained in the bubble undergoes an isothermal process. At large times the term $\mathrm{e}^{-\kappa^{*} r^{*}} / \sqrt{\pi r^{* *}}$ is generally small and the error function is close to unity. Therefore, equation (28) may be approximated as

$$
\frac{\mathrm{d} R^{*}}{\mathrm{~d} t^{*}}=-C_{0}^{*} \frac{1-C_{x}^{*}+\left(\sigma^{*} / R^{*}\right)}{1+\frac{1}{3}\left(\sigma^{*} / R^{*}\right)}\left(\frac{1}{R^{*}}+\sqrt{K^{*}}\right) .
$$

The solution of equation (29) can be obtained for small values of $K^{*}$ (such that $l / R^{*} \gg K^{*}$ ) as

$$
\begin{aligned}
1-\left(R^{*}\right)^{2} & -\frac{2 \sigma^{*}\left(1+2 C_{x}^{*}\right)\left(1-R^{*}\right)}{3\left(1-C_{\infty}^{*}\right)} \\
& +\frac{2\left(\sigma^{*}\right)\left(1+2 C_{\infty}^{* *}\right)}{3\left(1-C_{\infty}^{*}\right)^{2}} \ln \frac{\sigma^{*}+\left(1-C_{x}^{*}\right)}{\sigma^{*}+\left(1-C_{\infty}^{*}\right) R^{*}} \\
& =2 C_{\rho}^{*}\left(1-C_{\infty}^{*}\right) t^{*} .
\end{aligned}
$$

The resulting functions $R^{*}$ of equation (30) for $\sigma^{*}=1.4 \times 10^{-3}$ (corresponding to $\sigma=70$ dyn $/ \mathrm{cm}, \quad \bar{R}_{0}=0.1 \mathrm{~cm}$ and $p_{g n}=1.00140 \times$ $10^{4} \mathrm{dyn} / \mathrm{cm}^{2}$ ) are compared graphically in Fig. 4, with the corresponding solution of equation (30) without surface tension. i.e. $R^{*}=\left[1-2 C_{o}^{*}\left(1-C_{x}^{* *}\right) t^{*}\right]^{1 / 2}$. It is seen in the figure that the effect of surface tension is to shorten the time of complete solution. This can be explained by the bubble dynamics equation

$$
R \frac{\mathrm{d}^{2} R}{\mathrm{~d} t^{2}}+\frac{2}{3}\left(\frac{\mathrm{d} R}{\mathrm{~d} t}\right)^{2}=\frac{p_{g}-p(\infty)}{\rho}-\frac{2 \sigma}{\rho R}
$$

In the last equation, the surface tension term 


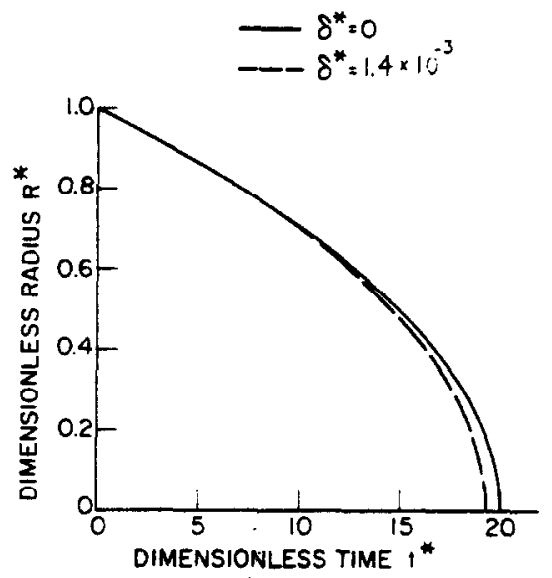

Fig. 4. Effect of surface tension on radius-time relation for dissolving bubbles in whole blood with low $K^{*}$ and plasma for $C_{p}^{*}=0.025$ and $C_{a}^{*}=0$.

has the same sign as the system-pressure term. indicating that the larger the surface tension force the faster will be the bubble collapse.

Finally, a bubble situated in a saturated solution will be examined. This corresponds to the special case $C_{x}^{*}=1$. If surface tension is neglected. equation (18) gives $R^{*}=1$. This implies that a bubble of any radius would be stable against diffusion in a saturated solution. with or without chemical reaction. However, in reality such a bubble dissolves due to surface tension. The solution of equation (29) with $C_{\propto}^{*}=1$ and neglect of the $\sqrt{K}$ term is

$1-\left(R^{*}\right)^{3}+\sigma^{*}\left[1-\left(R^{*}\right)^{2}\right]=3 \sigma^{*} C_{p}^{*} t^{*}$

Therefore, the time of complete solution is

$$
t^{*}=\frac{1+\sigma^{*}}{3 \sigma^{*} C_{\rho}^{*}}
$$

which decreases with an increase in surface tension $\sigma^{*}$.

Acknowledgement - This investigation is supported by a grant (grant number ! R01 HE12708) from the National Institute of Health for which the author is grateful.

\section{REFERENCES}

Dittmer. D. S. (Editor) (1961) Biological Handbooks Blood and other Body Fluids. Fedn Am. Soc. exp. Biology.

Epstein. P. S. and Plesset. M. S. (1950) On the stability of gas bubbles in liquid-gas solutions. $J$. chem. Phys 18. $1505-1509$.

Forster, R. E. (1964) Rate of gas uptake by red cells. Handbook of Physiology. (Section 3) Respiration (Edited by W. O. Fenn and H. Rahn). Chap. 32. p. 1.

Forster, R. E. and Van De Lindt. W. J. (1959) Calculations of the rates of uptake of $\mathrm{O}_{2}$ and $\mathrm{CO}$ by red cells using a digital compuier. Fedn Proc. 18. 47.

Gibson. Q. H. (1959) The kinetics of reactions between haemoglobin and gases. Progr. Biophys. Chem. 9. $1-53$.

Klug. A. Kreuzer. F. and Roughton. F. J. W. (1956) Simultaneous diffusion and chemical reaction in thin layers of haemoglobin solution. Proc. R. Soc. Lond. B145. 452-472.

Nicolson. P. and Roughton. F. J. W. (195)) A theore tical study of the influence of diffusion and chemical reaction velocity on the rate of exchange of carbon monoxide and oxygen between the red blood corpuscle and surrounding fiuid. Proc. $R$. Soc. Lond. B138. 241-264.

\section{NOMENCLATURE}

$C$ concentration of dissolved gas in liquid: $C_{.}$. under saturated state: $C_{x}$. at zero time or at a distance from bubble

$C^{*} \quad C / C_{s}: C_{*}^{*}=C_{*} / C_{s}$

$C_{p} \quad C_{s} / \rho_{0}$

$\therefore$ diffusion coefficient of dissolved gas in liquid

$K k^{\prime} \times$ (concentration of reduced hemoglobin)

$K^{*} \quad R_{0}{ }^{2} K / 5$

$k^{\prime}$ reaction velocity constant for the association of $\mathrm{O}_{2}$ and $\mathrm{Hb}$ to form $\mathrm{HbO}_{2}$

$M$ molecular weight of gas inside bubble

$m$ mass of gas inside bubble

$m^{*} \quad m / \rho_{n} R_{0}^{3}$

$n$ polytropic exponent

$p$ liquid pressure

$p_{g}$ gas pressure inside bubble: $p_{g \mid}$. at zero time

$R$ bubble radius: $R_{0}$, at zero time

$R^{*} \quad R / R_{0}$

$\bar{R}_{y} \quad$ universal gas constant

$r$ distance from the center of spherical bubble

$r / R_{0}$

$T$ liquid temperature or system temperature at zero time

I time.

\section{Greek letters}

$\alpha \rho^{*}+\sigma^{*} / R^{*}$

$\rho$ liquid density

$\rho_{\nu}$ density of gas inside bubble: $\rho_{g \pi \mid}$. at zero time: $\rho_{y x}$. under the same conditions of pressure and temperature with a gas-liquid surface of zero curvature

$\rho^{*} \quad \rho_{g \infty} / \rho_{\text {wi }}$

$\sigma$ surface tension

$\omega^{*} \quad 2 \sigma /\left(R_{0} p_{m}\right)$.

$$
\begin{array}{cl}
\text { Subscripts } \\
0 & t=0 \\
g & \text { gats } \\
s & \text { saturated value }
\end{array}
$$

